\title{
EXAMPLES OF FINSLER METRICS WITHOUT CONJUGATE POINTS: METRICS OF REVOLUTION
}

\author{
N. ZINOV'EV
}

\begin{abstract}
A large class of Finsler metrics without conjugate points on the torus that are far away from flat metrics is constructed.
\end{abstract}

\section{INTRODUCTION}

In accordance with the Hopf hypothesis (which was proved in [BuI]), all Riemannian metrics without conjugate points on the $n$-dimensional torus are flat. There are examples of nonflat Finsler metrics on tori without conjugate points. Here by a flat Finsler metric we mean a metric invariant under parallel translations, i.e., corresponding to some Minkowski norm on the universal cover of the torus.

Nevertheless, there is a conjecture that, in the Finslerian case, if there are no conjugate points, then the geodesic flow (on the unit tangent bundle) is conjugate to the flow of a flat Finsler metric. Not much is known about this, most likely due to the lack of examples.

It seems that the first examples of nonflat metrics without conjugate points on the two-dimensional torus were given in [Bu1] (see also [Bu2, §33]). Also, in [Bu1], there is a simple criterion for a system of curves on the two-dimensional torus to be realized as geodesics of some intrinsic metric without conjugate points. However, it is not quite clear under what conditions the resulting metric will be Finsler. There are examples, known to specialists but perhaps not published, of Finsler metrics without conjugate points on the torus that are obtained by perturbation of flat metrics.

In this paper we construct a large class of examples of Finsler metrics without conjugate points that are far away from flat ones. Moreover (see Theorem 1), a metric in question is determined essentially by an arbitrary one-parameter family of norms on $\mathbb{R}^{n}$ with fixed values on the hyperplane $\left\{y_{n}=0\right\}$. In contrast to the Riemannian case, a norm on the hyperplane admits significantly different (not affine-equivalent) extensions to the entire space.

Necessary information from Finsler geometry can be found, for example, in [Sh]. By a Finsler metric we mean a symmetric, smooth, and strictly convex Finsler structure on a smooth manifold $M$, i.e., a continuous function $\Phi: T M \rightarrow \mathbb{R}$ such that

(1) for every $x \in M$ the restriction $\Phi_{x}:=\left.\Phi\right|_{T_{x} M}$ is a Banach norm, in particular, $\Phi(v)=\Phi(-v)$ for all $v \in T M$;

(2) $\Phi$ is smooth on $T M \backslash 0$ (outside the zero cross section);

(3) for every $x \in M$ the matrix of the second derivatives of the function $\Phi_{x}^{2}$ is positive definite everywhere on $T_{x} M \backslash\{0\}$.

2000 Mathematics Subject Classification. Primary 53B40.

Key words and phrases. Hopf hypothesis, flat Finsler metrics, metric of revolution. 
These requirements ensure the existence of smooth geodesics and of the exponential map.

The length of a smooth curve $\gamma$ relative to a metric $\Phi$ is given by the formula

$$
\operatorname{Length}(\gamma)=\int \Phi(\dot{\gamma}(t)) d t
$$

The lengths of curves give rise to the distance function $d_{\Phi}$; namely, the distance between a pair of points is equal to the infimum of the lengths of piecewise smooth curves connecting them.

As in the Riemannian case, the concept of conjugate points can be introduced. It remains true that the absence of conjugate points is equivalent to the property that in the universal covering space every geodesic is minimal (i.e., it is the shortest curve between any two of its points).

In what follows we consider metrics on the $n$-dimensional torus $T^{n}=\mathbb{R}^{n} / \mathbb{Z}^{n}$, together with the corresponding lift of each metric to $\mathbb{R}^{n}$, the universal covering space of the torus. This lift is invariant with respect to the action of the group $\mathbb{Z}^{n}$ by parallel translations (which swap sheets of the covering). We denote a metric on the torus and its lift to $\mathbb{R}^{n}$ by the same letter $\Phi$.

We represent the $n$-dimensional torus as $T^{n}=T^{n-1} \times S^{1}$; by the standard action of the group $T^{n-1}$ on $T^{n}$ we mean its shift-action on the first factor. On the universal covering space, this corresponds to the action of the group $\mathbb{R}^{n-1} \times \mathbb{Z}$ by parallel translations.

Definition. We say that a Finsler metric on $T^{n}$ (or on $\mathbb{R}^{n}$ ) is a generalized metric of revolution if it is invariant under the standard action of the group $T^{n-1}$ (respectively, of $\left.\mathbb{R}^{n-1} \times \mathbb{Z}\right)$.

The first part of this paper is devoted to the proof of the following theorem.

Theorem 1. A generalized metric of revolution $\Phi$ on $T^{n}$ has no conjugate points if and only if, for every one-parameter subgroup $\left\{\psi^{t}\right\}$ of the group $T^{n-1}$, the vector field $X$ on $T^{n}$ given by the formula $X(p)=\left.\frac{\partial}{\partial t}\right|_{t=0} \psi^{t} p$ satisfies the condition $\Phi(X) \equiv$ const.

We state a coordinate version of this theorem for $\mathbb{R}^{n}$ viewed as the universal covering space of the torus. Let $\left(x_{1}, \ldots, x_{n}\right)$ be the standard coordinates in $\mathbb{R}^{n}$, and let $\left(y_{1}, \ldots, y_{n}\right)$ be the coordinates in the tangent spaces $T_{x} \mathbb{R}^{n}$ that correspond to the basis of coordinate vector fields $\left\{\frac{\partial}{\partial x_{1}}, \ldots, \frac{\partial}{\partial x_{n}}\right\}$. Thus, the tangent spaces at various points of $\mathbb{R}^{n}$ may be identified, and we can consider the Finsler metric $\Phi$ as the family $\left\{\Phi_{x}\right\}_{x \in \mathbb{R}^{n}}$ of norms on the space $\mathbb{R}^{n}=\left\{\left(y_{1}, \ldots, y_{n}\right)\right\}$.

Since $\Phi$ is a generalized metric of revolution, the norm $\Phi_{x}$ depends only on the $x_{n}$-coordinate of the point $x$, and this dependence is 1-periodic. Thus, the metric $\Phi$ is given by a one-parameter family of norms $\left\{\Phi_{t}\right\}_{t \in[0,1]}$, where $\Phi_{t}=\Phi_{(0, \ldots, 0, t)}$.

We put $H=\left\{\left(y_{1}, \ldots, y_{n}\right) \mid y_{n}=0\right\} \subset \mathbb{R}^{n}$. We say that the vectors in $H$ are horizontal, and call $H$ the horizontal subspace. The vector fields $X$ occurring in Theorem 1 correspond to constant horizontal vector fields on $\mathbb{R}^{n}$. Now, Theorem 1 takes the following form.

Theorem 1'. A generalized metric of revolution $\Phi$ has no conjugate points if and only if its horizontal sections are constant, i.e., in the above notation, $\left.\Phi_{x}\right|_{H}$ does not depend on $x$.

This theorem is proved in $\$ 2$. In the rest of the article we prove that the generalized metrics of revolution without conjugate points satisfy the above conjecture on conjugacy of geodesic flows.

For this, in 93 we show that the geodesic flow of a generalized metric of revolution without conjugate points has a complete set of independent $C^{\infty}$-smooth integrals. Given 
a rich isometry group, the presence of such integrals (multidimensional analogs of the Clairaut integral) follows from the Noether theorem. However, these integrals degenerate at horizontal directions; this is the main technical difficulty we have to cope with.

In $\$$ we calculate the stable norm of the metric (see 4 for the definition) and prove Theorem 2,

Theorem 2. If a generalized metric of revolution has no conjugate points, then its stable norm is $C^{\infty}$-smooth and quadratically strictly convex.

For a Finsler metric $\Phi$ on $T^{n}$, by $S_{\Phi} T^{n}$ we denote its unit tangent bundle, and by $g_{\Phi}^{t}: S_{\Phi} T^{n} \rightarrow S_{\Phi} T^{n}$ the geodesic flow on it. As usual, we say that the geodesic flows of Finsler metrics $\Phi$ and $\Psi$ are smoothly conjugate if there exists a diffeomorphism $h: S_{\Phi} T^{n} \rightarrow S_{\Psi} T^{n}$ such that $g_{\Phi}^{t}=h^{-1} \circ g_{\Psi}^{t} \circ h$ for all $t \in \mathbb{R}$.

Using standard techniques of Hamiltonian mechanics, in Subsection 4.1 we deduce the following statement from the results of $\S \$ 3$ and 4 .

Theorem 3. The geodesic flow of a generalized metric of revolution on the torus $T^{n}$ without conjugate points is smoothly conjugate to the flow of a flat Finsler metric on $T^{n}$ that corresponds to a stable norm.

Remark. A more general statement was mentioned in [CK] without proof: if the Heber foliation (see $[\mathrm{H}]$ ) of a Finsler metric without conjugate points on $T^{n}$ is smooth and regular, then its geodesic flow is smoothly conjugate to the geodesic flow of a flat Finsler torus. For metrics of revolution, the regularity of the Heber foliation can easily be deduced from Proposition 3.1, see also Remark 4.

The author is sincerely grateful to Sergei Ivanov and Dima Burago for suggesting the problem considered in this paper and for numerous helpful discussions.

\section{§1. Preliminaries}

Here we introduce some notation and list standard facts to be used further without explicit reference.

1.1. The Legendre transformation. Given a finite-dimensional vector space $V$, we denote by $V^{*}$ the dual space, i.e., the space of linear functions $V \rightarrow \mathbb{R}$. For a norm $\Psi$ on $V$, let $\Psi^{*}$ denote the dual norm on $V^{*}$, i.e.,

$$
\Psi^{*}(u)=\sup \{u(v): v \in V, \Psi(v)=1\} \quad \text { for } \quad u \in V^{*} .
$$

It is well known that if the norm $\Psi$ is smooth and quadratically strictly convex, then so is $\Psi^{*}$.

The second dual $V^{* *}$ is canonically identified with $V$; moreover, $\Psi^{* *}=\Psi$.

Given a Finsler metric $\Phi$ on a manifold $M$, we define a function $\Phi^{*}: T^{*} M \rightarrow \mathbb{R}$ by the rule $\left.\Phi^{*}\right|_{T_{x} M}=\left(\left.\Phi\right|_{T_{x} M}\right)^{*}, x \in M$, and introduce the sets

$$
\begin{aligned}
& S_{\Phi} M=\{v \in T M: \Phi(v)=1\}, \\
& S_{\Phi}^{*} M=\left\{u \in T^{*} M: \Phi^{*}(u)=1\right\},
\end{aligned}
$$

which are called the unit tangent and unit cotangent bundle of $\Phi$, respectively. It is not hard to check that $\Phi^{*}$ is a smooth function on $T^{*} M \backslash 0$, and $S_{\Phi} M, S_{\Phi}^{*} M$ are smooth submanifolds of $T M$ and $T^{*} M$.

Definition 1.1. Let $\Psi$ be a smooth quadratically strictly convex norm on a finitedimensional vector space $V$. The map

$$
\mathcal{L}_{\Psi}: V \rightarrow V^{*}
$$


defined by the formula

$$
\mathcal{L}_{\Psi}(v)=d_{v}\left(\frac{1}{2} \Psi^{2}\right), \quad v \in V,
$$

is called the Legendre transform of the norm $\Psi$. (Here, $d_{v}$ denotes the differential of a function at the point $v$. Note that, unlike $\Psi$, the function $\Psi^{2}$ is $C^{1}$-smooth everywhere on $V$, including zero.)

Obviously, the map $\mathcal{L}_{\Psi}$ is smooth and positive homogeneous on $V \backslash\{0\}$. It maps a point $v$ on the unit sphere of the norm $\Psi$ to the support linear function at $v$, i.e., $\Psi^{*}\left(\mathcal{L}_{\Psi}(v)\right)=1$ and $\mathcal{L}_{\Psi}(v)(v)=1$. Indeed, for every vector $w$ such that $\Psi(w)=1$, the function $g(t)=\Psi(v+w t)$ is 1-Lipschitz in $t$, whence $\left|\mathcal{L}_{\Psi}(v)(w)\right|=\Psi(v) \cdot\left|g^{\prime}(0)\right| \leq 1$, and equality is attained if and only if $w=v$. Similarly, the Legendre transform $\mathcal{L}_{\Psi^{*}}$ of the dual norm takes the resulting support function to the point $v$. Thus, the maps $\mathcal{L}_{\Psi}$ and $\mathcal{L}_{\Psi^{*}}$ are inverse of each other and map the unit spheres of the norms $\Psi$ and $\Psi^{*}$ to each other.

Definition 1.2. Let $M$ be a smooth manifold equipped with a Finsler metric $\Phi$. The map $\mathcal{L}_{\Phi}: T M \rightarrow T^{*} M$ that coincides on every fiber $T_{x} M$ with the Legendre transform of the norm $\left.\Phi\right|_{T_{x} M}$ is called the Legendre transform of the metric $\Phi$.

Clearly, the map $\mathcal{L}_{\Phi}$ is smooth and is a diffeomorphism between $T M \backslash 0$ and $T^{*} M \backslash 0$; furthermore, we have $\mathcal{L}_{\Phi}^{-1}=\mathcal{L}_{\Phi^{*}}$, where $\mathcal{L}_{\Phi^{*}}$ is a similar fiberwise Legendre transform of the cotangent bundle. Since $\mathcal{L}_{\Phi}$ takes the unit tangent vectors to unit covectors, we can view it as a diffeomorphism between $S_{\Phi} M$ and $S_{\Phi}^{*} M$.

If the metric $\Phi$ is fixed, we omit the index $\Phi$ in the notation for $\mathcal{L}_{\Phi}, S_{\Phi} M$, and $S_{\Phi}^{*} M$ (in particular, by $S \mathbb{R}^{n}$ and $S T^{n}$ we denote the unit tangent bundles of the metrics of revolution under consideration). For $x \in M$, we denote by $S_{x} M$ and $S_{x}^{*} M$ the fibers of the bundles $S M$ and $S^{*} M$ with the basepoint $x$.

1.2. Forms and calibrators. We need some notions related to differential 1-forms. We view a 1 -form $\omega$ on the manifold $M$ as a covector field on $M, \omega: M \rightarrow T^{*} M$, or as a fiberwise linear map $\omega: T M \rightarrow \mathbb{R}$. Therefore, for $x \in M, \omega(x)$ denotes a covector in $T_{x}^{*} M$, and for $v \in T_{x} M \subset T M$ the formula $\omega(v)$ denotes a number $\omega(x)(v) \in \mathbb{R}$.

The Legendre transform $\mathcal{L}$ of a Finsler metric sets up a bijective correspondence between the smooth vector fields and differential 1-forms. For a vector field $X$ on $M$, we denote by $\mathcal{L}(X)$ the differential form $\omega$ given by $\omega(x)=\mathcal{L}(X(x)), x \in M$. Similarly, the vector field $X$ can be represented as $\mathcal{L}^{-1}(\omega)$ for some differential form $\omega$.

Definition 1.3. Let $M$ be a smooth manifold with a Finsler metric $\Phi$, and let $\gamma$ be a geodesic of this metric parametrized naturally. A differential 1-form $\omega$ on $M$ is called a calibrating form (or a calibrator) for $\gamma$ if it is closed, $\Phi^{*}(\omega(x)) \leq 1$ for all $x \in M$, and $\omega(\dot{\gamma}(t))=1$ for all $t$.

The condition $\Phi^{*}(\omega(x)) \leq 1$ is equivalent to $|\omega(v)| \leq \Phi(v)$ for all $v \in T_{x} M$. If $\omega$ is a calibrator for $\gamma$ and $\omega(v)=1$ for some $v \in S_{x} M$, then $\omega(x)=\mathcal{L}(v)$, because $\mathcal{L}(v)$ is a unique support linear function for the norm $\Phi_{x}=\left.\Phi\right|_{T_{x} M}$ at the point $v$. Substituting $v=\dot{\gamma}(t)$, we see that $\omega(\gamma(t))=\mathcal{L}(\dot{\gamma}(t))$ for all $t$.

If a geodesic of a Finsler metric in $\mathbb{R}^{n}$ (more generally, in any simply connected manifold) has a calibrator, then this geodesic is minimal. Indeed, if $\omega$ is a calibrator of the geodesic segment $\gamma:[a, b] \rightarrow M$, then for every smooth curve $\gamma_{1}$ that connects the same pair of points we have $\int_{\gamma} \omega=\int_{\gamma_{1}} \omega$ because the form is closed. On the other hand, $\int_{\gamma_{1}} \omega \leq \operatorname{Length}\left(\gamma_{1}\right)$, with equality if and only if $\gamma_{1}=\gamma$, because $\omega\left(\dot{\gamma}_{1}\right) \leq \Phi\left(\dot{\gamma}_{1}\right)$ and $\omega(\dot{\gamma}) \equiv \Phi(\dot{\gamma})$. Hence, Length $(\gamma) \leq \operatorname{Length}\left(\gamma_{1}\right)$. 


\section{§2. Proof of Theorem 1}

Here we shall prove Theorem 1 , which is the coordinate version of Theorem 1, The "only if" part is contained in Lemma 2.2, the rest of this section is devoted to the proof of the "if" part.

We identify the tangent bundle $T \mathbb{R}^{n}$ with $\mathbb{R}^{n} \times \mathbb{R}^{n}$ and reserve the notation $x_{1}, \ldots$, $x_{n}$ for the coordinates of points of $\mathbb{R}^{n}$ and $y_{1}, \ldots, y_{n}$ for the coordinates of tangent vectors. Let $\Phi$ be a generalized metric of revolution on $\mathbb{R}^{n}$. By $\Phi_{x}$ we denote the norm on $\mathbb{R}^{n}=\left\{\left(y_{1}, \ldots, y_{n}\right)\right\}$ that corresponds to the restriction of $\Phi$ to $T_{x} \mathbb{R}^{n} \cong \mathbb{R}^{n}$.

Recall that $H$ denotes the subspace of horizontal vectors, $H=\left\{\left(y_{1}, \ldots, y_{n}\right) \mid y_{n}=0\right\}$. A tangent vector $(x, y) \in T_{x} \mathbb{R}^{n}$ is said to be horizontal if $y \in H$. We define a map $P_{H^{*}}: T^{*} \mathbb{R}^{n} \rightarrow H^{*}$ by the formula

$$
P_{H^{*}}(u)=\left.u\right|_{H}, \quad u \in T_{x}^{*} \mathbb{R}^{n} \subset T^{*} \mathbb{R}^{n},
$$

where $H$ is identified with the corresponding subspace of horizontal vectors in $T_{x} \mathbb{R}^{n} \cong$ $\mathbb{R}^{n}$. In coordinates, this map has the following representation:

$$
P_{H^{*}}(u)=\left(w_{1}, \ldots, w_{n-1}\right) \in \mathbb{R}^{n-1} \cong H^{*},
$$

where $\left(w_{1}, \ldots, w_{n-1}, w_{n}\right)$ are the coordinates of the covector $u$ in the standard basis of the subspace $T_{x}^{*} \mathbb{R}^{n}$.

Given a curve $\gamma=\gamma(t)$ in $\mathbb{R}^{n}$, we denote by $\gamma(t)_{n}$ its $n$-coordinate. Depending on the context, the velocity vector $\dot{\gamma}(t)$ will be regarded as a vector in $\mathbb{R}^{n}$ or in $T_{\gamma(t)} \mathbb{R}^{n} \subset T \mathbb{R}^{n}$.

Consider the Lagrangian $\phi=\frac{1}{2} \Phi^{2}: T \mathbb{R}^{n} \rightarrow \mathbb{R}$. The geodesic segments, parametrized proportionally to arc length, are extremals of the energy functional

$$
E(\gamma)=\int_{a}^{b} \phi(\gamma(t), \dot{\gamma}(t)) d t, \quad \gamma:[a, b] \rightarrow \mathbb{R}^{n}
$$

Consequently, they satisfy the system of Euler-Lagrange equations

$$
\frac{d}{d t} \frac{\partial \phi}{\partial y_{i}}(\gamma(t), \dot{\gamma}(t))=\frac{\partial \phi}{\partial x_{i}}(\gamma(t), \dot{\gamma}(t)), \quad i=1, \ldots, n .
$$

In our case, since $\frac{\partial \phi}{\partial x_{i}}=0$ for $i \neq n$, this system takes the form

$$
\left\{\begin{array}{l}
\frac{\partial \phi}{\partial y_{i}}(\gamma(t), \dot{\gamma}(t)) \equiv \text { const }=c_{i}, \\
\frac{d}{d t} \frac{\partial \phi}{\partial y_{n}}(\gamma(t), \dot{\gamma}(t))=\frac{\partial \phi}{\partial x_{n}}(\gamma(t), \dot{\gamma}(t)) .
\end{array}\right.
$$

By a horizontal line in a direction $h \in H$ we shall mean a curve of the form $\gamma(t)=$ $x^{0}+t h$, where $x^{0} \in \mathbb{R}^{n}$.

Lemma 2.1. A horizontal line $\gamma(t)=x^{0}+$ th is a geodesic if and only if $\frac{\partial \phi}{\partial x_{n}}\left(x^{0}, h\right)=0$.

Proof. For such a curve we have $\gamma(t)_{n}=$ const and $\dot{\gamma}(t)=h$. Hence, the first $n-1$ Euler-Lagrange equations

$$
\frac{\partial \phi}{\partial y_{i}}(\gamma(t), \dot{\gamma}(t)) \equiv \text { const }, \quad i=1, \ldots, n-1,
$$

are satisfied automatically. Since the metric is invariant under horizontal translations, the last equation takes the form

$$
\frac{\partial \phi}{\partial x_{n}}\left(x^{0}, h\right)=\frac{d}{d t} \frac{\partial \phi}{\partial y_{n}}\left(x^{0}, h\right)=0,
$$

as required.

Lemma 2.2. If a metric has no conjugate points, then $\left.\Phi_{x}\right|_{H}$ does not depend on $x$. 
Proof. Fixing a vector $h \in H$, we view $\Phi(x, h)$ as a function of the $x_{n}$-coordinate of the point $x \in \mathbb{R}^{n}$. Since this function is periodic, it attains its maximum $a_{\max }$ and minimum $a_{\text {min }}$. It suffices to prove that these two quantities are equal. Let $x^{0}$ and $x^{1}$ be the corresponding points of extremum, $\Phi\left(x^{0}, h\right)=a_{\min }, \Phi\left(x^{1}, h\right)=a_{\max }$. Then $\frac{\partial \phi}{\partial x_{n}}\left(x^{0}, h\right)=\frac{\partial \phi}{\partial x_{n}}\left(x^{1}, h\right)=0$, and by Lemma 2.1 the horizontal lines $t \mapsto x^{0}+t h$ and $t \mapsto x^{1}+t h$ are geodesics, hence minimal curves, because there are no conjugate points by assumption. The lengths of the segments between the corresponding points $x^{1}, x^{1}+t h$ and $x^{0}, x^{0}+t h$ are equal to $a_{\max } t$ and $a_{\min } t$, and by the triangle inequality we have

$$
\left|a_{\max } t-a_{\min } t\right| \leq d_{\Phi}\left(x^{0}, x^{1}\right)+d_{\Phi}\left(x^{0}+t h, x^{1}+t h\right)
$$

for all $t \in \mathbb{R}$. Since parallel translation by the vector $t h$ is an isometry, the right-hand side of this inequality does not depend on $t$ (and equals $2 d_{\Phi}\left(x^{0}, x^{1}\right)$ ). Hence, $a_{\max }=a_{\min }$.

Agreement. From now on in this section we assume that the restriction $\left.\Phi_{x}\right|_{H}$ does not depend on $x \in \mathbb{R}^{n}$. We denote this restriction by $\Phi_{H}$.

Lemma 2.3. All horizontal lines are geodesics. For any geodesic $\gamma$ that is not a horizontal line, we have $\operatorname{sgn} \dot{\gamma}(t)_{n}=$ const $\neq 0$.

Proof. The first statement follows from Lemma 2.1. The second follows from the first: if $\dot{\gamma}\left(t_{0}\right)_{n}=0$ for some $t_{0}$, then the uniqueness of a geodesic with given initial data shows that $\gamma$ coincides with the horizontal line in the direction of $\dot{\gamma}\left(t_{0}\right)$ passing through the point $\gamma\left(t_{0}\right)$.

Remark. Lemma 2.3 implies that the shortest curve between any two points from the same horizontal section cannot leave it. Therefore, horizontal lines are minimal curves (in particular, they contain no conjugate points).

Definition 2.4. The Clairaut integral of the metric $\Phi$ is defined to be the map $K$ : $T \mathbb{R}^{n} \backslash 0 \rightarrow H^{*} \cong \mathbb{R}^{n-1}$ introduced by the formula

$$
K(v)=P_{H^{*}}(\mathcal{L}(v)), \quad v \in T \mathbb{R}^{n} .
$$

The map $K$ is a multidimensional analog of the Clairaut integral of two-dimensional surfaces of revolution. Note that $K$ is invariant under parallel translations by the vectors from $\mathbb{R}^{n-1} \times \mathbb{Z}$.

We shall show that for every geodesic $\gamma$ the value of $K(\dot{\gamma})$ is constant along it. Indeed, in coordinates, $K(\dot{\gamma})$ has the form

$$
K(\dot{\gamma}(t))=\left(\mathcal{L}(\dot{\gamma}(t))\left(e_{1}\right), \ldots, \mathcal{L}(\dot{\gamma}(t))\left(e_{n-1}\right)\right),
$$

where the $e_{i}=\frac{\partial}{\partial x_{i}}$ are the standard basis vectors in $T_{\gamma(t)} \mathbb{R}^{n}$, and

$$
\mathcal{L}(\dot{\gamma}(t))\left(e_{i}\right)=\frac{\partial \phi}{\partial y_{i}}(\gamma(t), \dot{\gamma}(t)) \equiv c_{i}
$$

for $i<n$ by the Euler-Lagrange equations.

Remark. It is not hard to check that $K$ is the standard Noether integral corresponding to the action of the group $\mathbb{R}^{n-1}$.

Lemma 2.5. If $\gamma$ is a nonhorizontal naturally parametrized geodesic, then $\gamma(t)_{n}$ is a monotone function unbounded both from below and from above.

Proof. Monotonicity follows from Lemma 2.3. To prove unboundness, we show that for every interval $[a, b]$ the derivative $\dot{\gamma}(t)_{n}$ is separated away from zero whenever $\gamma(t)_{n} \in$ $[a, b]$. 
Observe that a vector $v \in S \mathbb{R}^{n}$ is horizontal if and only if $\left(\Phi_{H}\right)^{*}(K(v))=1$. Hence, two unit tangent vectors with the same value of $K$ are horizontal or not simultaneously. Let $K(\dot{\gamma}) \equiv w$; then the set

$$
\left\{v=(x, y) \in S \mathbb{R}^{n}: x_{1}=\cdots=x_{n-1}=0, x_{n} \in[a, b], \quad K(v)=w\right\}
$$

is compact and contains no horizontal vectors. Hence, the $y_{n}$-coordinates of the elements of this set are separated away from 0 . Consequently, $\dot{\gamma}(t)_{n}$ is separated away from 0 when $\gamma(t)_{n} \in[a, b]$.

Lemma 2.6. Every naturally parametrized geodesic $\gamma$ has a calibrating 1-form.

Proof. First, we construct a smooth vector field $X$ on $\mathbb{R}^{n}$ that is invariant under horizontal shifts and is such that $\Phi(X) \equiv 1, K(X)=$ const, and $X(\gamma(t))=\dot{\gamma}(t)$ for all $t$. If $\gamma$ is a horizontal line in the direction $h \in H$, we may take $X$ to be a constant vector field equal to $h$ at every point. Then the required properties follow from the fact that the restriction $\left.\Phi_{x}\right|_{H}=\Phi_{H}$ is constant. Indeed, for every $x \in \mathbb{R}^{n}$ we have $\Phi(x, h)=\Phi_{H}(h)=1$, and $K(x, h)=P_{H^{*}}(\mathcal{L}(x, h))$ is a (unique) supporting linear function of the norm $\Phi_{H}$ at the point $h$.

If $\gamma$ is not horizontal, then, by Lemma 2.5. the entire space is foliated by the geodesics that are horizontal translates of $\gamma$. We take $X$ to be equal to the velocity field of this foliation. The smoothness of this field follows from the fact that $\dot{\gamma}(t)_{n}$ does not vanish. Since the Clairaut integral $K(\dot{\gamma})$ is constant, it follows that $K(X) \equiv K(\dot{\gamma})=$ const.

Now we put $\omega=\mathcal{L}(X)$ and prove that $\omega$ is the calibrator we are looking for. The properties of the field $X$ imply that this 1-form $\omega$ is invariant under horizontal shifts, $\Phi^{*}(\omega) \equiv 1, \omega(\dot{\gamma}) \equiv 1$, and $P_{H^{*}}(\omega(x))=K(X(x))$ does not depend on $x \in \mathbb{R}^{n}$. So, we only need to prove that $\omega$ is closed. This follows from horizontal invariance and the fact that $P_{H^{*}}(\omega(x))$ is constant. Indeed, these properties imply that, in coordinates, $\omega$ has the form

$$
\omega(x)=c_{1} d x_{1}+\cdots+c_{n-1} d x_{n-1}+f(x) d x_{n},
$$

where $c_{1}, \ldots, c_{n-1}$ are some constants and $f(x)$ depends only on the $x_{n}$-coordinate. Then

$$
d \omega=\sum_{i \neq n} \frac{\partial f}{\partial x_{i}} d x_{i} \wedge d x_{n}=0 .
$$

Therefore, $\omega$ is a calibrating form for $\gamma$.

Proof of the theorem. The necessity of the condition $\left.\Phi_{x}\right|_{H} \equiv$ const is contained in Lemma 2.2. Conversely, if $\left.\Phi_{x}\right|_{H} \equiv$ const, then, by Lemma 2.6. every geodesic has a calibrator, whence every geodesic is minimal, and this is equivalent to the absence of conjugate points.

Remark. We have used the periodicity of the metric in $x_{n}$ only to derive the necessity of the condition $\left.\Phi_{x}\right|_{H} \equiv$ const (see Lemma 2.2).

\section{§3. Construction of a complete system of integrals of the Geodesic flow}

The Clairaut integral $K$ constructed in $\$ 2$ is a smooth integral of the geodesic flow of the generalized metric of revolution, but this integral degenerates on horizontal directions.

Our aim is to construct a smooth nondegenerate integral $\mathbb{K}$ for all directions; namely, we shall prove the following Proposition 3.1, which is the cornerstone of Theorem 3 .

Proposition 3.1. If a generalized metric of revolution on the $n$-dimensional torus $T^{n}$ has no conjugate points, then there exists a smooth map $\mathbb{K}: S T^{n} \rightarrow S^{n-1}$ that is an 
integral of the geodesic flow and has the following properties:

1) for all $p \in T^{n}$, the restriction $\left.\mathbb{K}\right|_{S_{p} T^{n}}: S_{p} T^{n} \rightarrow S^{n-1}$ is a diffeomorphism;

2) for all $w \in S^{n-1}$, the covector field $\mathcal{L}\left(\mathbb{K}^{-1}(w)\right)$ on $T^{n}$ is a graph of a closed 1-form on $T^{n}$.

Here, $S T^{n}$ denotes the unit tangent bundle, and $\mathcal{L}$ denotes the Legendre transform of the metric in question.

We shall derive Proposition 3.1 from Proposition 3.7 (see the end of Subsection 3.2), which is also used in $\$$. The map $\mathbb{K}$ will be constructed not for the metric on the torus, but for its lift to $\mathbb{R}^{n}$.

3.1. Notation. Let $\Phi$ be a generalized metric of revolution in $\mathbb{R}^{n}$ without conjugate points. We continue to use the notation introduced at the beginning of $\mathrm{g}_{2}$, as well as the notation $\Phi_{H}$ for the restriction $\left.\Phi_{x}\right|_{H}$ (which does not depend on $x \in \mathbb{R}^{n}$ by Theorem 1 ) and $K$ for the Clairaut integral $\left(K: S \mathbb{R}^{n} \rightarrow H^{*}, K(v)=P_{H^{*}} \mathcal{L}(v)\right.$, see Definition 2.4). Recall that $S \mathbb{R}^{n}$ and $S^{*} \mathbb{R}^{n}$ denote the unit (relative to the metric $\Phi$ ) tangent and cotangent bundle (respectively), and $\mathcal{L}$ is the Legendre transform of the metric $\Phi$.

We fix the notation $\left(w_{1}, \ldots, w_{n}\right)$ for the standard coordinates in the fibers of the cotangent bundle $T^{*} \mathbb{R}^{n}$ and identify $T^{*} \mathbb{R}^{n}$ with $\mathbb{R}^{n} \times \mathbb{R}^{n}=\{(x, w)\}$, where $x=\left(x_{1}, \ldots, x_{n}\right)$, $w=\left(w_{1}, \ldots, w_{n}\right)$. It is convenient to introduce the short notation $\bar{w}=\left(w_{1}, \ldots, w_{n-1}\right)$. Recall that in these coordinates the map $P_{H^{*}}: T^{*} \mathbb{R}^{n} \rightarrow H^{*} \cong \mathbb{R}^{n-1}$ has the form

$$
P_{H^{*}}\left(x, \bar{w}, w_{n}\right)=\bar{w} .
$$

Given a vector $v \in \mathbb{R}^{n-1} \times \mathbb{Z}$, we denote by $T^{v}$ the parallel translation by this vector, $T^{v}: \mathbb{R}^{n} \rightarrow \mathbb{R}^{n}$. Recall that $\Phi$ and $K$ are invariant under $T^{v}$.

3.2. Proof of Proposition 3.1. The following lemma shows that a vector $v \in S_{p} \mathbb{R}^{n}$ is uniquely determined by the value $K(v)$ of the Clairaut integral and by the nonstrict sign of its $y_{n}$-coordinate.

Lemma 3.2. Let $p \in \mathbb{R}^{n}$, and let $v_{1}, v_{2} \in S_{p} \mathbb{R}^{n}$ be such that $K\left(v_{1}\right)=K\left(v_{2}\right)$ and the $y_{n}$-coordinates of the vectors $v_{1}$ and $v_{2}$ are both nonpositive or both nonnegative. Then $v_{1}=v_{2}$.

Proof. Assume that $v_{1} \neq v_{2}$. Put $F_{i}=\mathcal{L}\left(v_{i}\right) \in T_{p}^{*} \mathbb{R}^{n}$ for $i=1,2$. By identifying $T_{p} \mathbb{R}^{n}$ with $\mathbb{R}^{n}$, we may view $F_{i}$ as a linear map $\mathbb{R}^{n} \rightarrow \mathbb{R}$. Recall that $K\left(v_{i}\right)=P_{H^{*}} \mathcal{L}\left(v_{i}\right)=\left.F_{i}\right|_{H}$, so that $\left.F_{1}\right|_{H}=\left.F_{2}\right|_{H}$. Consider the linear function $F=F_{1}-F_{2}: \mathbb{R}^{n} \rightarrow \mathbb{R}$. Since it is zero on the hyperplane $H$, the values $F\left(v_{1}\right)$ and $F\left(v_{2}\right)$ both lie (nonstrictly) on the same side of 0 , because the vectors $v_{1}$ and $v_{2}$ lie on the same side of $H$. But $F_{1}\left(v_{1}\right)=F_{2}\left(v_{2}\right)=1$, $F_{1}\left(v_{2}\right)<1$, and $F_{2}\left(v_{1}\right)<1$, because $F_{i}$ is the support linear function of the norm $\Phi_{p}$ at the point $v_{i}$. Consequently, $F\left(v_{1}\right)>0$ and $F\left(v_{2}\right)<0$, a contradiction.

Corollary 3.3. Assume that $p \in \mathbb{R}^{n}$ and $u_{1}, u_{2} \in S_{p}^{*} \mathbb{R}^{n}$ are such that $P_{H^{*}}\left(u_{1}\right)=$ $P_{H^{*}}\left(u_{2}\right)$ and the $y_{n}$-coordinates of the vectors $\mathcal{L}^{-1}\left(u_{1}\right)$ and $\mathcal{L}^{-1}\left(u_{2}\right)$ are both nonpositive or both nonnegative. Then $u_{1}=u_{2}$.

Definition 3.4. By a standard calibrator, we mean any closed 1 -form $\omega$ on $\mathbb{R}^{n}$ that possesses the following properties:

1) $\omega(x) \in S_{x}^{*} \mathbb{R}^{n}$ for all $x \in \mathbb{R}^{n}$;

2) $P_{H^{*}}(\omega(x))$ does not depend on $x \in \mathbb{R}^{n}$;

$3)$ the sign of the $y_{n}$-coordinate of the vector $\mathcal{L}^{-1}(\omega(x))$ does not depend on $x \in \mathbb{R}^{n}$.

We need the following refinement of Lemma 2.6. 
Lemma 3.5. For any $p \in \mathbb{R}^{n}$ and any $u \in S_{p}^{*} \mathbb{R}^{n}$, there exists a unique standard calibrator $\omega$ with $\omega(p)=u$. Moreover,

1) $\omega$ is invariant under horizontal translations by the vectors in $\mathbb{R}^{n-1} \times \mathbb{Z}$;

2) $\omega$ calibrates the geodesic $\gamma$ determined by the condition $\dot{\gamma}(0)=\mathcal{L}^{-1}(u)$.

Proof. Uniqueness is evident from Definition 3.4 and Corollary 3.3. We prove existence and the additional properties.

Let $\gamma$ be a geodesic with $\dot{\gamma}(0)=v$. We construct a calibrator $\omega$ for $\gamma$ as in the proof of Lemma 2.6. namely, $\omega=\mathcal{L}(X)$, where $X$ is the vector field on $\mathbb{R}^{n}$ with $\Phi(X) \equiv 1$, $X(p)=\dot{\gamma}(0)$, and $K(X)=$ const. These relations imply that $\Phi^{*}(\omega) \equiv 1, \omega(p)=u$, and $P_{H^{*}}(\omega)=K(X)=$ const. By the construction of the field $X$ (see the proof of Lemma 2.6. and also Lemma 2.3), the sign of its $y_{n}$-coordinate is constant. Thus, the last condition of Definition 3.4 is also satisfied.

Invariance under $\mathbb{R}^{n-1} \times \mathbb{Z}$ follows from Lemma 3.2 if $x=T^{v}\left(x^{\prime}\right)$ with $x, x^{\prime} \in$ $\mathbb{R}^{n}$ and $v \in \mathbb{R}^{n-1} \times \mathbb{Z}$, then the vectors $X(x)$ and $d T^{v}\left(X\left(x^{\prime}\right)\right)$ both lie on $S_{x} \mathbb{R}^{n}$ and have the same values of the Clairaut integral and the same signs of the last coordinate. Consequently, they are equal. Therefore, the field $X$ and, with it, the 1-form $\omega$, are invariant under $T^{v}$.

Definition 3.6. Lemma 3.5 shows that for every $x \in \mathbb{R}^{n}$ and every nonzero covector $u \in T_{x}^{*} \mathbb{R}^{n}$ there is a unique 1 -form $\omega$ on $\mathbb{R}^{n}$ proportional to a standard calibrator and such that $\omega(x)=u$. We denote this form by $\omega^{u}$.

For all $x, p \in \mathbb{R}^{n}$, we define a map

$$
r_{p, x}: T_{x}^{*} \mathbb{R}^{n} \backslash\{0\} \rightarrow T_{p}^{*} \mathbb{R}^{n} \backslash\{0\}
$$

by the formula $r_{p, x}(u)=\omega^{u}(p)$. Then we introduce the map

$$
r: \mathbb{R}^{n} \times\left(T^{*} \mathbb{R}^{n} \backslash 0\right) \rightarrow T^{*} \mathbb{R}^{n} \backslash 0
$$

such that $r(p, u)=r_{p, x}(u)$ for $u \in T_{x} \mathbb{R}^{n} \backslash\{0\} \subset T \mathbb{R}^{n} \backslash 0$.

The first property in Definition 3.4 implies that the image $r_{p, x}(u)$ of the unit covector $u \in S_{x}^{*} \mathbb{R}^{n}$ is also a unit covector. Therefore, we may regard $r_{p, x}$ as a map from $S_{x}^{*} \mathbb{R}^{n}$ to $S_{p}^{*} \mathbb{R}^{n}$.

Proposition 3.7. The map $r$ is smooth. For all $p, x \in \mathbb{R}^{n}$, the map $r_{p, x}$ is a diffeomorphism between $T_{x}^{*} \mathbb{R}^{n} \backslash\{0\}$ and $T_{p}^{*} \mathbb{R}^{n} \backslash\{0\}$; moreover, the restriction $\left.r_{p, x}\right|_{S_{x}^{*} \mathbb{R}^{n}}$ is a diffeomorphism between $S_{x}^{*} \mathbb{R}^{n}$ and $S_{p}^{*} \mathbb{R}^{n}$.

Remark. In fact, we shall prove that the map that takes a tangent vector at some point to the tangent vector at another point with the same value of the Clairaut integral and the same sign of the $y_{n}$-coordinate depends smoothly on its arguments.

Before we start proving Proposition 3.7, we deduce Proposition 3.1 from it. We fix $p \in \mathbb{R}^{n}$ and define a map $\mathbb{K}: S \mathbb{R}^{n} \rightarrow S_{p}^{*} \mathbb{R}^{n}$ by the formula

$$
\mathbb{K}(v)=r_{p, x}(\mathcal{L}(v)), \quad v \in S_{x} \mathbb{R}^{n} .
$$

By Proposition 3.7 the map $\mathbb{K}$ is smooth, and for every $x \in \mathbb{R}^{n}$ the restriction $\left.\mathbb{K}\right|_{S_{x} \mathbb{R}^{n}}$ is a diffeomorphism onto $S_{p}^{*} \mathbb{R}^{n} \cong S^{n-1}$. By construction, for every $u \in S_{p}^{*} \mathbb{R}^{n}$ the set $\mathcal{L}\left(\mathbb{K}^{-1}(u)\right)$ is a graph of a smooth closed form $\omega^{u}$.

We show that $\mathbb{K}$ is an integral of the geodesic flow. Let $\gamma$ be a geodesic parametrized naturally; we put $u(t)=\mathcal{L}(\dot{\gamma}(t)), \omega=\omega^{u(0)}$. By Lemma 3.5, 2), $\omega$ is a calibrator for $\gamma$. Hence, $\omega(\gamma(t))=\mathcal{L}(\dot{\gamma}(t))=u(t)$, so that $\omega^{u(t)}=\omega$ for all $t$. Hence, $\mathbb{K}(\dot{\gamma}(t))=$ $r_{p, \gamma(t)}(u(t))=\omega(p)=$ const; i.e., $\mathbb{K}$ is an integral of the geodesic flow. 
Lemma 3.5, 10 shows that $\mathbb{K}$ is invariant under integral parallel translations. Therefore, it is the lift of some map $S^{*} T^{n} \rightarrow S_{p}^{*} \mathbb{R}^{n} \cong S^{n-1}$ that satisfies Proposition 3.1.

Thus, Proposition 3.1 follows from Proposition 3.7 .

3.3. Proof of Proposition 3.7. Obviously, $r_{p, p}$ is the identity map, and $r_{p, q}=r_{p, x} \circ$ $r_{x, q}$ for any $p, q, x \in \mathbb{R}^{n}$. Hence, $r_{p, x}$ is a bijection between $T_{x}^{*} \mathbb{R}^{n} \backslash 0$ and $T_{p}^{*} \mathbb{R}^{n} \backslash 0$ and $r_{p, x}^{-1}=r_{x, p}$.

Now the second part of Proposition 3.7 follows from the first. Indeed, the smoothness of the map $r$ implies the smoothness of the restrictions $r_{p, x}$ and $r_{x, p}=r_{p, x}^{-1}$, so that $r_{p, x}$ is a diffeomorphism. Similarly, $\left.r_{p, x}\right|_{T_{x}^{*} \mathbb{R}^{n}}$ is a diffeomorphism between $S_{x}^{*} \mathbb{R}^{n}$ and $S_{p}^{*} \mathbb{R}^{n}$. Thus, it suffices to show the smoothness of the map $r$.

We split the set $T^{*} \mathbb{R}^{n}$ into three sets $M_{0}, M_{+}$, and $M_{-}$that consist of all covectors $u \in T^{*} \mathbb{R}^{n}$ such that the $y_{n}$-coordinate of the vector $\mathcal{L}^{-1}(u)$ is zero, positive, or negative, respectively.

Clearly, these three sets are cones: for any $u \in T^{*} \mathbb{R}^{n}$ and any $\alpha>0$ the covector $\alpha u$ belongs to the same set as $u$. The sets $M_{+}$and $M_{-}$are open and connected because so are the corresponding sets $\mathcal{L}^{-1}\left(M_{+}\right)$and $\mathcal{L}^{-1}\left(M_{-}\right)$.

In the following lemma we list all the properties of the map $r$ that will be needed in our proof.

Lemma 3.8. The map $r: \mathbb{R}^{n} \times\left(T^{*} \mathbb{R}^{n} \backslash 0\right) \rightarrow T^{*} \mathbb{R}^{n} \backslash 0$ is fiberwise homogeneous in the second argument. For every $p \in \mathbb{R}^{n}$ and $u \in T^{*} \mathbb{R}^{n} \backslash 0$, the following is true:

1) $r(p, u) \in T_{p}^{*} \mathbb{R}^{n}$

2) $\Phi^{*}(r(p, u))=\Phi^{*}(u)$;

3) $P_{H^{*}}(r(p, u))=P_{H^{*}}(u)$;

4) if $u \in M_{0}$, then $r(p, u) \in M_{0}$, and the same is true for $M_{+}$and $M_{-}$.

Proof. Homogeneity and property (1) follow from the definition of $r$. Given homogeneity, the remaining properties follow from the requirements of Definition 3.4 .

We have the following consequence of statements 10 and 3) in Lemma 3.8.

Corollary 3.9. In coordinates, the map $r$ has the form

$$
r\left(p, x, \bar{w}, w_{n}\right)=\left(p, \bar{w}, r_{n}\left(p, x, \bar{w}, w_{n}\right)\right), \quad p, x \in \mathbb{R}^{n}, \quad \bar{w} \in \mathbb{R}^{n-1}, \quad w_{n} \in \mathbb{R},
$$

where $r_{n}$ is some function defined for $\left(\bar{w}, w_{n}\right) \neq 0$.

Lemma 3.10. The map $r$ is continuous.

Proof. By homogeneity, it suffices to prove that the restriction of $r$ to $\mathbb{R}^{n} \times S^{*} \mathbb{R}^{n}$ is continuous.

Assume that the covectors $u_{i} \in S^{*} \mathbb{R}^{n}, i=1,2, \ldots$, converge to $u \in S^{*} \mathbb{R}^{n}$, and points $p_{i} \in \mathbb{R}^{n}$ converge to a point $p$. We must show that the covectors $r\left(p_{i}, u_{i}\right)$ converge to $r(p, u)$. It suffices to check that for every point of condensation $\alpha$ of the sequence $\left\{r\left(p_{i}, u_{i}\right)\right\}$ we have $\alpha=r(p, u)$.

Lemma 3.8, 10, 22) shows that $r(p, u) \in S_{p}^{*} \mathbb{R}^{n}$ and $r\left(p_{i}, u_{i}\right) \in S_{p_{i}}^{*} \mathbb{R}^{n}$ for every $i$, whence $\alpha \in S_{p}^{*} \mathbb{R}^{n}$. By Lemma 3.8, 3), we have $P_{H^{*}}(r(p, u))=P_{H^{*}}(u)$ and $P_{H^{*}}\left(r\left(p_{i}, u_{i}\right)\right)=$ $P_{H^{*}}\left(u_{i}\right)$; passing to the limit, we get $P_{H^{*}}(\alpha)=P_{H^{*}}(r(p, u))$. Switching to a subsequence, we may assume that all the covectors $u_{i}$ lie in one of the sets $M_{0}, M_{+}$and $M_{-}$, and without loss of generality we may assume that all $u_{i}$ lie in $M_{0} \cup M_{+}$. Then $u \in M_{0} \cup M_{+}$ because the set $M_{0} \cup M_{+}$is closed. By Lemma 3.8, 4), $r(p, u)$ and $r\left(p_{i}, u_{i}\right)$ belong to $M_{0} \cup M_{+}$for every $i$, whence $\alpha \in M_{0} \cup M_{+}$. Since the covectors $\alpha$ and $r(p, u)$ satisfy the assumptions of Corollary 3.3. they are equal. 
We denote $\Psi=\Phi^{*}, \Psi_{x}=\left.\Psi\right|_{T_{x}^{*} \mathbb{R}^{n}}$. We view $\Psi$ as a function of the coordinates $\left(x, \bar{w}, w_{n}\right)$, where $x \in \mathbb{R}^{n}, \bar{w} \in \mathbb{R}^{n-1}, w_{n} \in \mathbb{R}$.

Let $\psi$ denote the norm on $H^{*}$ dual to $\Phi_{H}$.

Lemma 3.11. The sets $M_{0}, M_{+}$, and $M_{-}$have the following properties:

1) $\psi\left(P_{H^{*}}(u)\right) \leq \Psi(u)$ for all $u \in T^{*} \mathbb{R}^{n}$, with equality if and only if $u \in M_{0}$;

2) in coordinates, $M_{0}$ can be described as follows:

$$
M_{0}=\left\{(x, w) \in T^{*} \mathbb{R}^{n}: \frac{\partial \Psi^{2}}{\partial w_{n}}(x, w)=0\right\} ;
$$

3) $M_{0}$ can be represented as the graph

$$
M_{0}=\left\{\left(x, \bar{w}, w_{n}\right) \in T^{*} \mathbb{R}^{n}: w_{n}=f(x, \bar{w})\right\}
$$

of a function $f=f(x, \bar{w}), x \in \mathbb{R}^{n}, \bar{w} \in \mathbb{R}^{n-1}$, which is smooth outside the set $\{\bar{w}=0\}$;

4) $M_{+}$and $M_{-}$are the supergraph and subgraph of this function $f$ :

$$
M_{ \pm}=\left\{\left(x, \bar{w}, w_{n}\right) \in T^{*} \mathbb{R}^{n}: \operatorname{sgn}\left(w_{n}-f(x, \bar{w})\right)= \pm 1\right\} .
$$

Proof. 11) Suppose $u \in T_{x}^{*} \mathbb{R}^{n}, x \in \mathbb{R}^{n}$. By definition, $\Psi(u)$ is the maximal value of the covector $u$ applied to all vectors from $S_{x} \mathbb{R}^{n}$, and $\psi\left(P_{H^{*}}(u)\right)$ equals the maximal value of $u$ on the horizontal vectors in $S_{x} \mathbb{R}^{n}$. Therefore, $\psi\left(P_{H^{*}}(u)\right) \leq \Psi(u)$ and equality occurs if and only if the maximum of $u$ on $S_{x} \mathbb{R}^{n}$ is attained on some horizontal vector $h$, which means that $u$ is proportional to a covector from $\mathcal{L}(h) \in M_{0}$.

2) Recall that $\mathcal{L}^{-1}=\mathcal{L}_{\Psi}$ is the fiberwise Legendre transform of the family of norms $\Psi_{x}, x \in \mathbb{R}^{n}$. By the definition of the Legendre transform, the $y_{n}$-coordinate of the vector $\mathcal{L}_{\Psi}(u)$, where $u=(x, w) \in T^{*} \mathbb{R}^{n}$, is equal to $\frac{1}{2} \frac{\partial \Psi^{2}}{\partial w_{n}}(u)$. Consequently, $u \in M_{0}$ if and only if $\frac{\partial \Psi^{2}}{\partial w_{n}}(u)=0$.

Similarly, $u \in M_{+}$when $\frac{\partial \Psi^{2}}{\partial w_{n}}(u)>0$, and $u \in M_{-}$when $\frac{\partial \Psi^{2}}{\partial w_{n}}(u)<0$.

3) Fix $x \in \mathbb{R}^{n}$ and $\bar{w} \in \mathbb{R}^{n-1}$ and consider the function $g(t)=\Psi^{2}(x, \bar{w}, t)$. By property 1), the covector $u=\left(x, \bar{w}, w_{n}\right)$ belongs to $M_{0}$ if and only if $w_{n}$ is a point of minimum of the function $g$. Since $\Psi_{x}$ is a strictly convex norm, the function $g$ is strictly convex, and $g(t) \rightarrow+\infty$ as $t \rightarrow \pm \infty$, its minimum is attained at a unique point. Hence, $M_{0}$ is the graph $w_{n}=f(x, \bar{w})$ of some function $f$.

The smoothness of $f$ (for $\bar{w} \neq 0$ ) follows from the implicit function theorem. Indeed, by property (2), $M_{0}$ is given by the equation $\frac{\partial \Psi^{2}}{\partial w_{n}}=0$. This equation satisfies the conditions of the implicit function theorem (in the variable $w_{n}$ ): we have $\frac{\partial^{2} \Psi^{2}}{\partial w_{n}^{2}}>0$ because the norms $\Psi_{x}$ are quadratically strictly convex.

4) The function $g$ is strictly convex (see the proof of the preceding property). Hence, its derivative near the point of minimum is positive. Consequently, $\frac{\partial \Psi^{2}}{\partial w_{n}}\left(x, \bar{w}, w_{n}\right)>0$ whenever $w_{n}>f(x, \bar{w})$. The inequality $\frac{\partial \Psi^{2}}{\partial w_{n}}\left(x, \bar{w}, w_{n}\right)>0$ is equivalent to the fact that $\left(x, \bar{w}, w_{n}\right) \in M_{+}$; see the end of the proof of property 21). Thus, $\left(x, \bar{w}, w_{n}\right) \in M_{+}$ whenever $w_{n}>f(x, \bar{w})$. Similarly, $\left(x, \bar{w}, w_{n}\right) \in M_{-}$whenever $w_{n}<f(x, \bar{w})$.

Lemma 3.12. Outside of the set $\{\bar{w}=0\}$, the function $\Psi^{2}=\Psi^{2}\left(x, \bar{w}, w_{n}\right)$ can be represented in the form

$$
\Psi^{2}\left(x, \bar{w}, w_{n}\right)=\psi^{2}(\bar{w})+\left(w_{n}-f(x, \bar{w})\right)^{2} g\left(x, \bar{w}, w_{n}\right)^{2},
$$

where $f$ is the function occurring in Lemma (3.11), and the function $g=g\left(x, \bar{w}, w_{n}\right)$ is defined for $\bar{w} \neq 0$ and is smooth and strictly positive.

We shall use the following standard fact. 
Lemma 3.13. Let $U$ be an open set in $\mathbb{R}^{k}$ and assume that a function $h=h(x, y) \in$ $C^{\infty}(U \times \mathbb{R})$ satisfies $h(x, 0) \equiv 0$. Then there exists a function $h_{1} \in C^{\infty}(U \times \mathbb{R})$ such that

$$
h(x, y)=y \cdot h_{1}(x, y), \quad x \in U, y \in \mathbb{R} .
$$

If, moreover, it is known that $\frac{\partial h}{\partial y}(x, 0) \equiv 0$, then $h$ can be represented in the form

$$
h(x, y)=y^{2} \cdot g(x, y) \text {, where } g \in C^{\infty}(U \times \mathbb{R}) .
$$

Proof. By the Newton-Leibniz formula we have

$$
h(x, y)=\int_{0}^{1} \frac{d h(x, t y)}{d t} d t=\int_{0}^{1} y \cdot \frac{\partial h}{\partial y}(x, t y) d t,
$$

so that $h(x, y)=y \cdot h_{1}(x, y)$, where

$$
h_{1}(x, y)=\int_{0}^{1} \frac{\partial h}{\partial y}(x, t y) d t .
$$

This yields a smooth function $h_{1}$ on $U \times \mathbb{R}$. The first statement is proved.

Note that $h_{1}(x, 0)=\frac{\partial h}{\partial y}(x, 0)$. If this expression is equal to zero everywhere, then we can apply the first part of the lemma to $h_{1}$ in place of $f$, and the second statement follows.

Proof of Lemma 3.12, By Lemma 3.11,

$$
\Psi(x, \bar{w}, f(x, \bar{w}))=\psi(\bar{w}), \quad \frac{\partial \Psi^{2}}{\partial w_{n}}(x, \bar{w}, f(x, \bar{w})) \equiv 0
$$

for all $x \in \mathbb{R}^{n}, \bar{w} \in \mathbb{R}^{n-1} \backslash\{0\}, w_{n} \in \mathbb{R}$. Applying Lemma 3.13 to the function

$$
h\left(x, \bar{w}, w_{n}\right)=\Psi^{2}\left(x, \bar{w}, w_{n}+f(x, \bar{w})\right)-\psi^{2}(\bar{w})
$$

on the set $\mathbb{R}^{n} \times\left(\mathbb{R}^{n-1} \backslash\{0\}\right) \times \mathbb{R}$, we get the representation

$$
\Psi^{2}\left(x, \bar{w}, w_{n}\right)=\psi^{2}(\bar{w})+\left(w_{n}-f(x, \bar{w})\right)^{2} g\left(x, \bar{w}, w_{n}\right),
$$

where $x \in \mathbb{R}^{n}, \bar{w} \in \mathbb{R}^{n-1} \backslash\{0\}, w_{n} \in \mathbb{R}$, and $g$ is a smooth function. If $\left(x, \bar{w}, w_{n}\right) \notin M_{0}$, then $\Psi\left(x, \bar{w}, w_{n}\right)>\psi(\bar{w})$ by Lemma 3.11, 1), whence $g\left(x, \bar{w}, w_{n}\right)>0$. If $\left(x, \bar{w}, w_{n}\right) \in M_{0}$, i.e., if $w_{n}=f(x, \bar{w})$, we have

$$
g\left(x, \bar{w}, w_{n}\right)=\frac{1}{2} \frac{\partial^{2} \Psi^{2}}{\partial w_{n}^{2}}\left(x, \bar{w}, w_{n}\right)>0,
$$

because $\Psi_{x}$ is a quadratically strictly convex norm. Thus, the function $g$ is positive everywhere. Redenoting $\sqrt{g}$ by $g$, we get the required statement.

Now we are in a position to prove Proposition 3.7. As was already noted above, it suffices to check the smoothness of the map $r$. By Corollary [3.9, in coordinates the map $r$ has the form

$$
r\left(p, x, \bar{w}, w_{n}\right)=\left(p, \bar{w}, r_{n}\left(p, x, \bar{w}, w_{n}\right)\right)
$$

for $p, x \in \mathbb{R}^{n},\left(\bar{w}, w_{n}\right) \in \mathbb{R}^{n} \backslash\{0\}$. Thus, it suffices to prove that the coordinate function $r_{n}$ is smooth.

Lemma 3.8, 21) implies that $r_{n}$ satisfies the equation

$$
\Psi\left(p, \bar{w}, r_{n}\left(p, x, \bar{w}, w_{n}\right)\right)=\Psi\left(x, \bar{w}, w_{n}\right) .
$$

By Lemma 3.11, 21), outside the set $M_{0}$ we have $\frac{\partial \Psi}{\partial w_{n}} \neq 0$; hence, we can apply the implicit function theorem to (3.1) and $r_{n}$. Since the function $r_{n}$ is continuous (see Lemma 3.10), the implicit function theorem shows that it is smooth in a neighborhood of every point 
$\left(p, x, \bar{w}, w_{n}\right)$ such that $r\left(p, x, \bar{w}, w_{n}\right)=\left(p, \bar{w}, r_{n}\left(p, x, \bar{w}, w_{n}\right)\right) \notin M_{0}$. Consequently, $r_{n}$ is smooth on the set

$$
r^{-1}\left(T^{*} \mathbb{R}^{n} \backslash M_{0}\right)=\mathbb{R}^{n} \times\left(T^{*} \mathbb{R}^{n} \backslash M_{0}\right) .
$$

It remains to prove that $r_{n}$ is smooth in some neighborhood of the set $r^{-1}\left(M_{0} \backslash 0\right)=$ $\mathbb{R}^{n} \times\left(M_{0} \backslash 0\right)$. Note that $\bar{w} \neq 0$ for all points $\left(p, x, \bar{w}, w_{n}\right)$ of this set.

Squaring both sides of (3.1) and substituting the expression for $\Phi^{2}$ from Lemma 3.12. we get

$$
\begin{aligned}
\left(r_{n}-f(p, \bar{w})\right)^{2} g\left(p, \bar{w}, r_{n}\right)^{2} & =\left(w_{n}-f(x, \bar{w})\right)^{2} g\left(x, \bar{w}, w_{n}\right)^{2}, \\
r_{n} & =r_{n}\left(p, x, \bar{w}, w_{n}\right) .
\end{aligned}
$$

Lemmas 3.8, 44) and 3.11, (4) show that the map $r$ satisfies the condition

$$
\operatorname{sgn}\left(r_{n}\left(p, x, \bar{w}, w_{n}\right)-f(p, \bar{w})\right)=\operatorname{sgn}\left(w_{n}-f(x, \bar{w})\right)
$$

for all $p, x \in \mathbb{R}^{n}, \bar{w} \in \mathbb{R}^{n-1} \backslash\{0\}, w_{n} \in \mathbb{R}$. Using this condition and the fact that $g$ is positive, from (3.2) we deduce that

$$
F\left(p, \bar{w}, r_{n}\left(p, x, \bar{w}, w_{n}\right)\right)=F\left(x, \bar{w}, w_{n}\right),
$$

where

$$
F\left(x, \bar{w}, w_{n}\right)=\left(w_{n}-f(x, \bar{w})\right) g\left(x, \bar{w}, w_{n}\right) .
$$

By Lemma 3.11, 3), for $\left(p, \bar{w}, r_{n}\left(p, x, \bar{w}, w_{n}\right)\right) \in M_{0} \backslash 0$, we have $r_{n}\left(p, x, \bar{w}, w_{n}\right)=f(p, \bar{w})$. For a point $\left(x, \bar{w}, w_{n}\right)$ such that $\bar{w} \neq 0$ and $w_{n}=f(x, \bar{w})$, we have $\frac{\partial F}{\partial w_{n}}\left(x, \bar{w}, w_{n}\right)=$ $g\left(x, \bar{w}, w_{n}\right)>0$. Hence, we can apply the implicit function theorem to (3.3) viewed as an equation for $r_{n}$. Since the function $r_{n}$ is continuous (see Lemma 3.10), this shows that it is smooth in some neighborhood of every point of the set $r^{-1}\left(M_{0} \backslash 0\right)$.

The proof of Proposition 3.7, and, with it, of Proposition 3.1, is finished.

\section{$\S 4$. Calculation of the stable norm}

In our case, it is quite convenient to use the definition of the stable norm in terms of the universal covering space of the $n$-dimensional torus (see also [BBI]). The standard definition can be found, e.g., in Ban.

Let $\Phi$ be an arbitrary $\mathbb{Z}^{n}$-periodic Finsler metric on $\mathbb{R}^{n}$, and let $d=d_{\Phi}$ be the corresponding intrinsic metric. It is known Bur that the metric space $\left(\mathbb{R}^{n}, d\right)$ is at a finite Gromov-Hausdorff distance from some normed space. More precisely, there exists a norm $\|\cdot\|_{\text {st }}$, called the stable norm of the metric, and a constant $C$ such that for any $x, y \in \mathbb{R}^{n},\left|d(x, y)-\|x-y\|_{\mathrm{st}}\right|<C$.

Remark. In the case where the metric has no conjugate points, this statement is much easier to prove than in the general case. Namely, it is not hard to show (see, e.g., [Bu2, §32]) that for every $v \in \mathbb{Z}^{n}$ the distance $d(x, x+v)$ does not depend on $x \in \mathbb{R}^{n}$ and $d(x, x+k v)=k d(x, x+v)$ for every positive integer $k$ and $x \in \mathbb{R}^{n}$. Using these properties, one can put $\|v\|_{\mathrm{st}}=d(x, x+v)$ for $v \in \mathbb{Z}^{n}$ and extend $\|\cdot\|_{\mathrm{st}}$ as a homogeneous continuous function to the whole of $\mathbb{R}^{n}$.

From the definition it is clear that for any vector $v \in \mathbb{R}^{n}$ we have

$$
\|v\|_{s t}=\lim _{t \rightarrow+\infty} \frac{d(p, p+t v)}{t}
$$

for any point $p \in \mathbb{R}^{n}$.

In this section, we calculate the stable norm of the generalized metric of revolution without conjugate points (see Corollary 4.8) and prove Theorem 2 i.e., we show that the stable norm of such a metric is smooth and quadratically strictly convex. 
Below in this section, $\Phi$ denotes a generalized metric of revolution in $\mathbb{R}^{n}$, and $d=d_{\Phi}$ is the corresponding intrinsic metric. We continue to use the notation fixed in the preceding sections.

Lemma 4.1. For any $x \in \mathbb{R}^{n}$ and any $v \in \mathbb{R}^{n-1} \times \mathbb{Z}$, the geodesic $\gamma$ connecting the points $x$ and $x+v$ is invariant under the parallel translation $T^{v}$ by the vector $v$.

Proof. Let $x=\gamma(0), x+v=\gamma(a)$. We use the fact that the Clairaut integral $K(\dot{\gamma}(t))$ and $\operatorname{sgn} \dot{\gamma}(t)_{n}$ are constant (see Lemma 2.3). By Lemma 3.2. the velocity vector $\dot{\gamma}(a)$ is uniquely determined by the values $K(\dot{\gamma}(a))$ and $\operatorname{sgn} \dot{\gamma}_{n}(a)$. For the vector $d T^{v} \dot{\gamma}(0)$, these parameters have the same values; hence, $\dot{\gamma}(a)=d T^{v} \dot{\gamma}(0)$, which means that the geodesic is $T^{v}$-invariant.

Remark. The lemma also follows from the fact that the function $f(x)=d\left(x, T^{v}(x)\right)$ is constant (see Remark 4). Indeed, let $\gamma$ be the geodesic segment between $x$ and $x+v$, and let $q$ be its midpoint; then $d\left(q, T^{v}(q)\right)=\operatorname{Length}(\gamma)$. Hence, $\gamma \cup T^{v}(\gamma)$ is a geodesic.

Lemma 4.2. If $v \in \mathbb{R}^{n-1} \times \mathbb{Z}$, then $\|v\|_{\mathrm{st}}=d(p, p+v)$ for all $p \in \mathbb{R}^{n}$. The restriction of the norm $\|\cdot\|_{\text {st }}$ to the horizontal subspace $H$ coincides with $\Phi_{H}$.

Proof. By Lemma 4.1, the shift $T^{v}$ takes the geodesic $\gamma$ through $p$ and $p+v$ into itself. Hence, this geodesic goes through all the points of the form $p+n v$ with $n \in \mathbb{N}$. Since there are no conjugate points, we see that $\gamma$ is minimal; consequently, $d(p, p+n v)=n d(p, p+v)$. Therefore, $\|v\|_{\text {st }}=\lim \frac{d(p, p+n v)}{n}=d(p, p+v)$. If $v \in H$, then $d(p, p+v)=\Phi_{H}(v)$, because $\gamma$ is a horizontal line (see Lemma 2.3).

Remark. It is easy to deduce the strict convexity of the stable norm from Lemma 4.2 , i.e., to prove that there are no segments on its unit sphere.

Indeed, if there is a segment on the unit sphere of the stable norm, then $\|v+u\|_{\text {st }}=$ $\|v\|_{\text {st }}+\|u\|_{\text {st }}$ for some noncollinear vectors $u, v \in \mathbb{R}^{n-1} \times \mathbb{Z}$. Hence, by Lemma 4.2, we have $d(0, v+u)=d(0, v)+d(v, v+u)$; i.e., the triangle inequality turns into equality for the points $0, v, v+u$, which, by Lemma 4.1. do not lie on the same geodesic.

Recall that for any $x \in \mathbb{R}^{n}$ and any nonzero covector $u \in T_{x}^{*} \mathbb{R}^{n}$, there exists a unique 1 -form $\omega^{u}$ on $\mathbb{R}^{n}$ proportional to a standard calibrator and such that $\omega^{u}(x)=u$ (see Definition 3.6). By Definition 3.4 and Lemma 3.5, 1), $\omega^{u}$ is a closed $\mathbb{Z}^{n}$-periodic 1-form on $\mathbb{R}^{n}$, so that it determines an element $\left[\omega^{u}\right]$ of the group $H^{1}\left(T^{n} ; \mathbb{R}\right)$ of the one-dimensional de Rham cohomologies of the torus $T^{n}=\mathbb{R}^{n} / \mathbb{Z}^{n}$.

We define a positive homogeneous map

$$
I: T^{*} \mathbb{R}^{n} \backslash 0 \rightarrow H^{1}\left(T^{n} ; \mathbb{R}\right)=\mathbb{R}^{n *}
$$

by the formula $I(u)=\left[\omega^{u}\right]$. We identify $H^{1}\left(T^{n} ; \mathbb{R}\right)$ with $\mathbb{R}^{n *}$, by using the standard isomorphism $H_{1}\left(T^{n}\right) \cong \mathbb{Z}^{n}$.

Lemma 4.3. Suppose $u \in T^{*} \mathbb{R}^{n} \backslash 0, p \in \mathbb{R}^{n}, v \in \mathbb{R}^{n-1} \times \mathbb{Z}$, and $\gamma$ is a smooth curve connecting $p$ and $p+v$. Then $I(u)(v)=\int_{\gamma} \omega^{u}$.

Proof. For $v \in \mathbb{Z}^{n}$, the formula follows from the definition of $I(u)$, because the projection of the curve $\gamma$ to $T^{n}$ represents an element of the homology group that corresponds to the vector $v$. In the general case, for every natural $k$ we consider the curve $\gamma^{k}=$ $\bigcup_{i=0}^{k-1}\left(T^{v}\right)^{i} \gamma$ connecting $p$ and $p+k v$. Since the form $\omega^{u}$ is $T^{v}$-invariant (by Lemma 3.5), we have $\int_{\gamma^{k}} \omega^{u}=k \int_{\gamma} \omega^{u}$. On the other hand, the differences $\left|\int_{\gamma^{k}} \omega^{u}-I(u)(k v)\right|$ for $k \in \mathbb{N}$ are uniformly bounded, because the vectors $k v$ lie on a bounded distance from the integral lattice. Since $\left|\int_{\gamma^{k}} \omega^{u}-I(u)(k v)\right|=k\left|\int_{\gamma} \omega^{u}-I(u)(v)\right|$, this implies that $\int_{\gamma} \omega^{u}-I(u)(v)=0$ 
Lemma 4.4. For every $p \in \mathbb{R}^{n}$ and $v \in \mathbb{R}^{n-1} \times \mathbb{Z}$, we have

$$
\|v\|_{\mathrm{st}}=\sup _{u \in S_{p}^{*} \mathbb{R}^{n}} I(u)(v) .
$$

The supremum is attained precisely at one covector $u \in S_{p}^{*} \mathbb{R}^{n}, u=\mathcal{L}(\dot{\gamma}(0))$, where $\gamma$ is a naturally parametrized geodesic connecting the points $p$ and $p+v, \gamma(0)=p$.

Proof. Let $u \in S_{p}^{*} \mathbb{R}^{n}$, and let $\gamma$ be a geodesic segment between $p$ and $p+v$. By the preceding lemma, we have $I(u)(v)=\int_{\gamma} \omega^{u}$. The properties of calibrators (see Subsection 1.2) show that $d(p, p+v) \geq \int_{\gamma} \omega^{u}$, with equality attained if and only if $\omega^{u}$ calibrates $\gamma$, i.e., if and only if $u=\mathcal{L}(\dot{\gamma}(0))$ (see Lemma 3.5). Therefore, $d(p, p+v)=\sup _{u \in S_{p}^{*} \mathbb{R}^{n}} I(u)(v)$. By Lemma 4.2, $d(p, p+v)=\|v\|_{\mathrm{st}}$, which completes the proof.

Below, $\partial B_{s t}^{*}$ denotes the unit sphere of the norm $\|\cdot\|_{\text {st }}^{*}$ dual to the stable norm; $t e_{n}$ denotes the point in $\mathbb{R}^{n}$ with coordinates $(0, \ldots, 0, t)$. This notation is used till the end of the section.

Lemma 4.5. For $p \in \mathbb{R}^{n}$, let $\left(w_{1}, \ldots, w_{n}\right)$ be the coordinates of the covector $u \in$ $T_{p}^{*} \mathbb{R}^{n} \backslash\{0\}$ relative to the standard basis of the space $T_{p}^{*} \mathbb{R}^{n}$. Then

$$
I(u)=\left(w_{1}, \ldots, w_{n-1}, \int_{0}^{1} r_{n}\left(t e_{n}, p, \bar{w}, w_{n}\right) d t\right),
$$

where $\bar{w}=\left(w_{1}, \ldots, w_{n-1}\right)$, and $r_{n}$ is a coordinate function of the map $r$ (see Definition 3.6 and Corollary 3.9]).

Proof. Definition 3.6 and Corollary 3.9 show that, in coordinates, the form $\omega^{u}$ looks like this:

$$
\omega^{u}(x)=w_{1} d x_{1}+\cdots+w_{n-1} d x_{n-1}+r_{n}\left(x, p, \bar{w}, w_{n}\right) d x_{n} .
$$

By Lemma 4.3, we have $I_{i}(u)=I(u)\left(e_{i}\right)=\int_{\gamma_{i}} \omega^{u}$, where the curve $\gamma_{i}:[0,1] \rightarrow \mathbb{R}^{n}$ is defined by $\gamma_{i}(t)=t e_{i}$. (Here $I_{i}$ is the $i$ th coordinate function of the map $I, i=1, \ldots, n$.) Substituting the coordinate expression for $\omega^{u}$ in the integral $\int_{\gamma_{i}} \omega^{u}$, we get the required statement.

Lemma 4.6. The map I is smooth. For every $p \in \mathbb{R}^{n}$, the differential of the restriction $\left.I\right|_{T_{p}^{*} \mathbb{R}^{n} \backslash\{0\}}$ is nondegenerate everywhere.

Proof. Smoothness follows from formula (4.2) and from the smoothness of the function $r_{n}$ (see Proposition 3.7).

Since for all $x \in \mathbb{R}^{n}$ the map $r_{x, p}: T_{p}^{*} \mathbb{R}^{n} \backslash\{0\} \rightarrow T_{x}^{*} \mathbb{R}^{n} \backslash\{0\}$ is a diffeomorphism (see Proposition 3.7) and does not change the coordinates $w_{1}, \ldots, w_{n-1}$ (see Corollary 3.9), we have $\frac{\partial r_{n}}{\partial w_{n}} \neq 0$. Since the domain of the map in question is connected, the sign of the derivative $\frac{\partial r_{n}}{\partial w_{n}}$ is constant, and since $r_{p, p}$ is the identity map, this sign is positive. Together with (4.2), this implies that $\frac{\partial I_{n}}{\partial w_{n}}>0$. Consequently, since $I$ also keeps the coordinates $w_{1}, \ldots, w_{n-1}$, the differential of $\left.I\right|_{T_{p}^{*} \mathbb{R}^{n}}$ is nondegenerate.

Proposition 4.7. For any $p \in \mathbb{R}^{n}$, the restriction $\left.I\right|_{S_{p}^{*} \mathbb{R}^{n}}$ is a diffeomorphism onto $\partial B_{\mathrm{st}}^{*}$. In particular, $\partial B_{\mathrm{st}}^{*}$ is a smooth submanifold of $\mathbb{R}^{n *}$.

Proof. Let $u \in S_{p}^{*} \mathbb{R}^{n}$. By Lemma 4.4 $I(u)(v) \leq\|v\|_{\text {st }}$ for any $v \in \mathbb{R}^{n-1} \times \mathbb{Z}$, and hence, for any $v \in \mathbb{R}^{n}$. Moreover, equality is attained (at a vector $v \in \mathbb{R}^{n-1} \times \mathbb{Z}$ such that the geodesic $\gamma$ with the initial velocity vector equal to $\mathcal{L}^{-1}(u)$ connects $p$ and $p+v$; such a geodesic exists by Lemma 2.5). Consequently, $I(u) \in \partial B_{\mathrm{st}}^{*}$.

Let $v \in \mathbb{R}^{n-1} \times \mathbb{Z}$ be such that $I(u)(v)=\|v\|_{\mathrm{st}}, u^{\prime} \in S_{p}^{*} \mathbb{R}^{n}, u^{\prime} \neq u$. Then $I\left(u^{\prime}\right)(v)<$ $\|v\|_{\mathrm{st}}$ by Lemma 4.4, whence $I\left(u^{\prime}\right) \neq I(u)$. Thus, the restriction $\left.I\right|_{S_{p}^{*} \mathbb{R}^{n}}$ is injective. By 
Lemma 4.6, this restriction is smooth and its differential is nondegenerate. Hence, it is a diffeomorphism onto a smooth submanifold $I\left(S_{p}^{*} \mathbb{R}^{n}\right) \subset \mathbb{R}^{n *}$, which is contained in $\partial B_{\mathrm{st}}^{*}$. Since $\partial B_{\text {st }}^{*}$ is homeomorphic to $S^{n-1}$ and any continuous injective map from $S^{n-1}$ to $S^{n-1}$ is surjective, the image $I\left(S_{p}^{*} \mathbb{R}^{n}\right)$ coincides with $\partial B_{\mathrm{st}}^{*}$.

Corollary 4.8. The unit sphere of the dual stable norm can be represented in the form

$$
\partial B_{s t}^{*}=\left\{I(w) \mid w \in S^{*} \mathbb{R}^{n}\right\}=\{[\omega] \mid \omega \text { is a standard calibrator }\} .
$$

Proposition 4.9. The hypersurface $\partial B_{\mathrm{st}}^{*}$ is quadratically convex; i.e., for every point $u \in \partial B_{\mathrm{st}}^{*}$ there exists a Euclidean ball $B$ such that $u \in \partial B$ and $\partial B_{\mathrm{st}}^{*} \subset B$.

Proof. For $x \in \mathbb{R}^{n}$, we denote by $S_{x}^{*}$ the hypersurface in $\mathbb{R}^{n *}$ that corresponds to the cotangent sphere $S_{x}^{*} \mathbb{R}^{n}$ under the identification $T_{x}^{*} \mathbb{R}^{n} \cong \mathbb{R}^{n *}$. We fix the notation $\left(w_{1}, \ldots, w_{n}\right)$ for coordinates in $\mathbb{R}^{n *}$. As before, we denote $\bar{w}=\left(w_{1}, \ldots, w_{n-1}\right)$.

Fix a point $p \in \mathbb{R}^{n}$. By Proposition 3.7 and Corollary 3.9, the hypersurface $S_{x}^{*} \subset \mathbb{R}^{n *}$ can be parametrized by a diffeomorphism $r_{x, p}: S_{p}^{*} \rightarrow S_{x}^{*}$, which in coordinates has the form

$$
r_{x, p}\left(\bar{w}, w_{n}\right)=\left(\bar{w}, r_{n}\left(x, p, \bar{w}, w_{n}\right)\right)
$$

(we keep the notation $r_{x, p}$ for the map described in Definition 3.6 composed with the identifications $T_{x}^{*} \mathbb{R}^{n} \cong T_{p}^{*} \mathbb{R}^{n} \cong \mathbb{R}^{n *}$ ). By Proposition 4.7 and Lemma 4.5, the hypersurface $\partial B_{\text {st }}^{*} \subset \mathbb{R}^{n *}$ can be parametrized by a diffeomorphism $I_{p}: S_{p}^{*} \rightarrow \partial B_{\mathrm{st}}^{*}$, where

$$
I_{p}(w)=\left(\bar{w}, \int_{0}^{1} r_{n}\left(t e_{n}, p, \bar{w}, w_{n}\right) d t\right)=\int_{0}^{1} r_{t e_{n}, p}(w) d t
$$

for $w=\left(\bar{w}, w_{n}\right) \in S_{p}^{*}$.

Fix $w^{0}=\left(\bar{w}^{0}, w_{n}^{0}\right) \in S_{p}^{*}$. We shall show that the hypersurface $\partial B_{\text {st }}^{*}$ is quadratically strictly convex at the point $u^{0}=I_{p}\left(w^{0}\right)$. We consider two cases.

Case 1. $\mathcal{L}^{-1}\left(p, w^{0}\right)$ is a horizontal vector. This means that the covector $\left(p, w_{0}\right)$ and all the covectors of the form $\left(x, r_{x, p}\left(w^{0}\right)\right)$ lie in the set $M_{0}$ (see Proposition 3.3 and Lemma 3.8(4) ).

Since the hypersurfaces $S_{x}^{*}$ are quadratically strictly convex, for every $x \in \mathbb{R}^{n}$ there exists a Euclidean ball $B_{x}$ containing $S_{x}^{*}$ and such that $r_{x, p}\left(w^{0}\right) \in \partial B_{x}$. By compactness, we may assume that the radii of all the balls $B_{x}$ are the same.

Note that all the tangent hyperplanes to the surfaces $S_{x}^{*}$ at the points $r_{x, p}\left(w^{0}\right)$ coincide. Indeed, items 1) and 2) of Lemma 3.11 show that these hyperplanes are parallel to the coordinate axis $w_{n}$, and their images under the projection $P_{H^{*}}$ touch the unit ball of the norm $\psi=\left(\Phi_{H}\right)^{*}$ at the point $\bar{w}^{0}$ from $H^{*} \cong \mathbb{R}^{n-1}$.

Hence, all the balls $B_{x}$ can be identified by parallel translations that interchange the points $r_{x, p}\left(w^{0}\right)$. Let $B$ denote the ball obtained from $B_{x}$ by a parallel translation that takes $r_{x, p}\left(w^{0}\right)$ to $u^{0}$. We shall show that $\partial B_{\text {st }}^{*} \subset B$.

For every $w \in S_{p}^{*}$ and $x \in \mathbb{R}^{n}$ we have $r_{x, p}(w) \in B_{x}$. Thus, by the construction of the ball $B$, the point $c(x, w)=u^{0}+r_{x, p}(w)-r_{x, p}\left(w^{0}\right)$ lies in $B$. From formula (4.4), it follows that the point $I_{p}(w)=u^{0}+I_{p}(w)-I_{p}\left(w^{0}\right)$ lies in the convex hull of the points $c\left(t e_{n}, w\right), t \in[0,1]$, whence $I_{p}(w) \in B$. Since $I_{p}\left(S_{p}^{*}\right)=\partial B_{\mathrm{st}}^{*}$, this means that $\partial B_{s t}^{*} \subset B$.

Case 2. $\mathcal{L}^{-1}\left(p, w^{0}\right)$ is not horizontal. It suffices to prove that the second fundamental form of the hypersurface $\partial B_{\mathrm{st}}^{*}$ is either positive definite, or negative definite at the point $u^{0}$. 
Since $\frac{\partial \Psi}{\partial w_{n}}\left(p, w^{0}\right) \neq 0$ (see item 2 of Lemma 3.11), some neighborhood $U$ of the point $w^{0}$ in $S_{p}^{*}$ can be represented as the graph

$$
U=\left\{(\bar{w}, h(\bar{w})) \mid \bar{w} \in U^{0}\right\}
$$

of a smooth function $h: U^{0} \rightarrow \mathbb{R}$, where $U^{0}$ is some neighborhood of the point $\bar{w}^{0}$ in $\mathbb{R}^{n-1}$.

Now, from (4.3) it follows that for any $x \in \mathbb{R}^{n}$ the corresponding domain $r_{x, p}(U) \subset S_{x}^{*}$ is represented as a graph:

$$
r_{x, p}(U)=\left\{\left(\bar{w}, g_{x}(\bar{w})\right) \mid \bar{w} \in U^{0}\right\},
$$

where $g_{x}(\bar{w})=r_{n}(x, p, \bar{w}, h(\bar{w}))$.

Formula (4.4) shows that the corresponding neighborhood $I_{p}(U)$ of the point $u^{0}$ in $\partial B_{\mathrm{st}}^{*}$ can be represented as the graph of a smooth function $g: U^{0} \rightarrow \mathbb{R}$, where

$$
g(\bar{w})=\int_{0}^{1} g_{t e_{n}}(\bar{w}) d t, \quad \bar{w} \in U^{0} .
$$

We use the following simple fact: if a hyperplane $N \subset \mathbb{R}^{n}$ can be represented locally as the graph of a smooth function $g: \mathbb{R}^{n-1} \rightarrow \mathbb{R}$, then its second fundamental form has a definite sign if and only if so does the second derivative $d^{2} g$ (i.e., the quadratic form with the coefficients $\frac{\partial^{2} g}{\partial u_{i} \partial u_{j}}$ ).

Since every surface $S_{x}^{*}$ is quadratically strictly convex, the quadratic form $d^{2} g_{x}\left(\bar{w}^{0}\right)$ has a definite sign. Moreover, by continuity, its sign is the same for all $x \in \mathbb{R}^{n}$. Therefore, the quadratic form $d^{2} g\left(\bar{w}^{0}\right)=\int_{0}^{1} d^{2} g_{t e_{n}}\left(\bar{w}^{0}\right) d t$ also has a definite sign, so that the hypersurface $\partial B_{\mathrm{st}}^{*}$ is quadratically strictly convex at the point $u^{0}$.

Proof of Theorem 2, Propositions 4.7 and 4.9 show that the dual stable norm is infinitely smooth and quadratically strictly convex. Hence, so is $\|\cdot\|_{\text {st }}$.

Remark. By the limit direction of a geodesic $\gamma$ of a Finsler metric in $\mathbb{R}^{n}$ we mean the limit $D(\gamma)=\lim _{t \rightarrow \infty} \frac{\gamma(t)}{t}$ if it exists. In the case of a generalized metric of revolution, this limit always exists because $\gamma$ is invariant under the shift $T^{v}$ by some vector $v \in \mathbb{R}^{n-1} \times \mathbb{Z}$ (see Lemma 4.11).

Let $\mathcal{L}_{\mathrm{st}}: \partial B_{\mathrm{st}} \rightarrow \partial B_{\mathrm{st}}^{*}$ be the Legendre transform of the stable norm. It is easily seen that for any $v \in S \mathbb{R}^{n}$ the vector $\mathcal{L}_{\text {st }}^{-1}(I(\mathcal{L}(v)))$ coincides with $D\left(\gamma_{v}\right)$, where $\gamma$ is the geodesic with $\dot{\gamma}_{v}(0)=v$. The maps $I \circ \mathcal{L}$ and $\mathcal{L}_{\text {st }}^{-1} \circ I \circ \mathcal{L}$ have all the properties of the map $\mathbb{K}$ listed in Proposition 3.1. Thus, the constructed integral of the geodesic flow acquires a clear geometric sense.

Remark. It is not hard to show that the fibers $I^{-1}(y), y \in \mathbb{R}^{n *}$, coincide with the fibers of the foliation constructed in $[\mathrm{H}$. The latter ones are the graphs of the differentials of the Busemann functions. In $[\mathrm{H}]$ it was shown that if the metric $\Phi$ has no conjugate points, then for $w \in S \mathbb{R}^{n}$ the Busemann function $b_{w}$ of the geodesic $\gamma$ with $\dot{\gamma}(0)=w$ coincides with $-b_{-w}$. In other words, for all $x \in \mathbb{R}^{n}$ we have

$$
b_{w}(x)=\lim _{t \rightarrow \infty} d_{\Phi}(x, \gamma(t))-t=\lim _{t \rightarrow \infty} t-d_{\Phi}(x, \gamma(-t)) .
$$

From this, it is not hard to deduce that an arbitrary 1-Lipshitz function $g$ such that $g(\gamma(t))=t$ for all $t \in \mathbb{R}$ coincides with $b_{-w}$. Therefore, every standard calibrator is a differential of some Busemann function. 
4.1. Proof of Theorem 3, We regard $T^{*} T^{n}$ as a symplectic manifold with the standard symplectic form $\omega$. Since the map $I$ is invariant under integral parallel translations, it is a lift of some map $F: T^{*} T^{n} \backslash 0 \rightarrow \mathbb{R}^{n *}$. For every $y \in \mathbb{R}^{n *} \backslash\{0\}$, the fiber $F^{-1}(y)$ is the graph of some closed 1-form $\omega^{y}$ (this form lifts to $\mathbb{R}^{n}$ as $\omega^{u}$, where $u \in I^{-1}(y)$ ). This means that $F^{-1}(y)$ is a Lagrange submanifold (see [TF, $\S 24$; Fom, $\left.\S 3.1\right]$ ).

This and Theorem 35.2 in [DNF, Part 1] imply that the coordinate functions of the map $F$ are in involution (see also Proposition 15 in TF, §24] and the study of Lagrange fibrations in TF, §28]). Consequently, we can apply the Liouville theorem (see Arn, $\mathrm{DNF}, \mathrm{BF}$ ) that states the existence of "action-angle" coordinates in a neighborhood of every torus $F^{-1}(y)$. Namely, for every point $y_{0} \in \mathbb{R}^{n *}$ there exists a neighborhood $U \ni y_{0}$ and smooth functions $s_{1}, \ldots, s_{n}: F^{-1}(U) \rightarrow \mathbb{R}$ (action coordinates) and $\varphi_{1}, \ldots, \varphi_{n}$ : $F^{-1}(U) \rightarrow \mathbb{R} / \mathbb{Z}$ (angle coordinates) such that

1) the functions $s_{i}$ are constant on every fiber $F^{-1}(y), y \in U$;

2) the map $\left(s_{1}, \ldots, s_{n}, \varphi_{1}, \ldots, \varphi_{n}\right)$ is a diffeomorphism from $F^{-1}(U)$ onto $V \times T^{n}$, where $V$ is an open domain in $\mathbb{R}^{n}$;

3) $\omega=\sum_{i} d s_{i} \wedge d \varphi_{i}$.

Moreover, the action coordinates can be obtained in the following way. Assume that, on every torus $F^{-1}(y), y \in U$, we have fixed a set $\gamma_{1, y}, \ldots, \gamma_{n, y}$ of cycles representing the basis of the fundamental group $\pi_{1}\left(F^{-1}(y)\right) \cong \mathbb{Z}^{n}$ and that $\gamma_{i, y}$ depends on $y$ continuously. Let $\alpha$ be a 1 -form on $F^{-1}(U)$ such that $d \alpha=\omega$. Then we can define $s_{i}$ by an explicit formula (see $[\mathrm{BF}, \S 1.5])$ :

$$
s_{i}(u)=\int_{\gamma_{i, y}} \alpha, \quad \text { where } u \in F^{-1}(U), y=F(u) .
$$

Let $\gamma_{1}, \ldots, \gamma_{n}$ be some cycles in $T^{n}$ that represent the standard basis of the fundamental group $\pi_{1}\left(T^{n}\right) \cong \mathbb{Z}^{n}$. We select $\gamma_{i, y}$ to be the cycle in $F^{-1}(y)$ that projects to $\gamma_{i}$ under the projection map of the bundle $\pi: T^{*} M \rightarrow M$. Let $\alpha$ be the canonical action form (given by $\alpha(a)=\xi(d \pi(a))$, where $\left.a \in T_{(x, \xi)} T^{*} M\right)$.

Let $y \in U, u \in F^{-1}(y)$. Recall that $F^{-1}(y)$ is the graph of a smooth 1 -form $\omega^{y}$, and that $y=F(u)=\left[\omega^{y}\right]$ is an element of the cohomology group $H^{1}\left(T^{n}, \mathbb{R}\right) \cong \mathbb{R}^{n *}$, which corresponds to the form $\omega^{y}$. Now the formula for $s_{i}$ takes the form

$$
s_{i}(u)=\int_{\gamma_{i, y}} \alpha=\int_{\gamma_{i}} \omega^{y}=\left[\omega^{y}\right]\left(e_{i}\right)=F_{i}(u),
$$

where the $F_{i}$ are the coordinate functions of the map $F$. Thus, the action coordinates coincide with the coordinate functions of the map $F$. In particular, they are defined everywhere on $T^{*} T^{n} \backslash 0$. The choice of the origin on every torus $F^{-1}(y), y \in \mathbb{R}^{n *} \backslash\{0\}$, determines the angle coordinates uniquely. Thus, the action-angle coordinates can be defined globally as

$$
(s, \varphi): T^{*} T^{n} \backslash 0 \rightarrow\left(\mathbb{R}^{n *} \backslash\{0\}\right) \times T^{n},
$$

and $\|s(u)\|_{\mathrm{st}}^{*}=\Phi^{*}(u), u \in T^{*} T^{n} \backslash 0$ (see Proposition 4.7).

Let $\Phi_{0}$ be a flat Finsler metric on $T^{n}$ that corresponds to the stable norm of the metric $\Phi$ (here we use Theorem 2). We construct similar coordinates $\left(s^{0}, \varphi^{0}\right)$ for the metric $\Phi_{0}$ and define the diffeomorphism $h: T^{*} T^{n} \backslash 0 \rightarrow T^{*} T^{n} \backslash 0$ by $h=\left(s^{0}, \varphi^{0}\right)^{-1} \circ(s, \varphi)$. This diffeomorphism keeps the symplectic structure and satisfies $H=H_{0} \circ h$, where $H$ and $H_{0}$ are Hamiltonians of the metrics $\Phi$ and $\Phi_{0}$, respectively.

Therefore, $h$ conjugates the Hamiltonian flows of the metrics $\Phi$ and $\Phi_{0}$, as well as their restrictions to the unit cotangent bundles. Since the Legendre transformation maps the geodesic flow to the Hamiltonian flow, the geodesic flows of the metrics $\Phi$ and $\Phi_{0}$ are also conjugate. 


\section{REFERENCES}

[Arn] V. I. Arnol'd, Mathematical methods of classical mechanics, "Nauka", Moscow, 1989; English transl. of the 1974 Russian original, Grad. Texts in Math., vol. 60, Springer-Verlag, New York, 1992. MR1037020 (93c:70001) MR1345386 (96c:70001)

[Ban] V. Bangert, Minimal geodesics, Ergodic Theory Dynam. Systems 10 (1989), 263-286. MR.1062758 (91j:58126)

[BBI] D. Yu. Burago, Yu. D. Burago, and S. V. Ivanov, A course in metric geometry, Moscow-Izhevsk, 2004; English transl., Grad. Stud. in Math., vol. 33, Amer. Math. Soc., Providence, RI, 2001. MR.1835418 (2002e:53053)

[Bu1] H. Busemann, Metrics on the torus without conjugate points, Bol. Soc. Mat. Mexicana 10 (1953), nos. 3-4, 1-18. MR0065225 (16:399f)

[Bu2] — The geometry of geodesics, Acad. Press, Inc., New York, NY, 1955. MR0075623 $(17: 779 \mathrm{a})$

[Bur] D. Burago, Periodic metrics, Adv. Soviet Math., vol. 9, Amer. Math. Soc., Providence, RI, 1992, pp. 205-210. MR 1166203 (93c:53029)

[BuI] D. Burago and S. Ivanov, Riemannian tori without conjugate points are flat, Geom. Funct. Anal. 4 (1994), no. 3, 259-269. MR1274115 (95h:53049)

[BF] A. V. Bolsinov and A. T. Fomenko, Integrable Hamiltonian systems. Geometry, topology, classification, Udmurt. Univ., Izhevsk, 1999; English transl., Chapman and Hall/CRC, Boca Raton, FL, 2004. MR1792190(2002g:37071) MR2036760 (2004j:37106)

[CK] C. B. Croke and B. Kleiner, On tori without conjugate points, Invent. Math. 120 (1995), 241-257. MR:1329041 (96j:53037)

[DNF] B. A. Dubrovin, S. P. Novikov, and A. T. Fomenko, Modern geometry - methods and applications. Parts I-III, 5th ed., "Editorial URSS", Moscow, 2001; English transl. of 1st ed., Part I, Grad. Texts in Math., vol. 93, Springer-Verlag, New York, 1984; Part II, Grad. Texts in Math., vol. 104, Springer-Verlag, New York, 1985; Part III, Grad. Texts in Math., vol. 124, Springer-Verlag, New York, 1990. MR0736837 (85a:53003) MR0807945 (86m:53001) MR:1076994 (91j:55001)

[Fom] A. T. Fomenko, Symplectic geometry, Moskov. Gos. Univ., Moscow, 1988; English transl., Adv. Stud. in Contemp. Math., vol. 5, Gordon and Breach Sci. Publ., New York, 1988. MR0964470 (90k:58082) MR0994805 (90k:58065)

[H] Jens Heber, On the geodesic flow of tori without conjugate points, Math. Z. 216 (1994), 209-216. MR.1278421 (95d:58104)

[Sh] Z. Shen, Lectures on Finsler geometry, World Sci. Publ. Co., Singapore, 2001. MR1845637 (2002f:53032)

[TF] V. V. Trofimov and A. T. Fomenko, Algebra and geometry of integrable Hamiltonian differential equations, Mathematics and its Applications, "Faktorial", Moscow; copublished with Udmurt. Univ., Izhevsk, 1995. (Russian) MR1469742 (98f:58118)

Sun Microsystems SPB, LlC, 10th Krasnoarmeyskaya Street, 22-A, St. Petersburg, 190103, Russia

E-mail address: nikita.zinoviev@gmail.com

Received 8/FEB/2008

Translated by THE AUTHOR 